\title{
Commercial Radar Sensors and Applications
}

\author{
Thilo Lenhard \\ InnoSenT GmbH, Am Roedertor 30, D-97499 Donnersdorf \\ thilo.lenhard@innosent.de
}

\begin{abstract}
:
The rapidly increasing automation - e.g. the developments towards autonomous driving and the progression of Industry 4.0 - implicates growing demands on presence and motion detection for higher safety. Due to the availability of affordable high frequency components, novel packaging solutions and advanced production processes Radar sensors are no longer associated only with huge and expensive systems. They are low cost solutions for measurement tasks, which cannot be covered satisfactorily with optical or acoustic/ultrasound systems. Nowadays Radar sensors are omnipresent and can be found in many situations of everyday life, where they might not be expected. This paper describes various applications for commercial microwave sensors, explains specific challenges and shows individual solutions regarding Radar hardware, modulation techniques and signal processing.
\end{abstract}

Key words: Radar Sensor, Industry 4.0, Modulation, Radar Signal Processing, Resolution

\section{Introduction}

The benefits of radar sensors are evident: Radar is capable of measuring speed, distance and the angle of arrival as well as the direction of movement. Radar can work in adverse conditions like rain, fog and dust and is able to cover long range as well as close distance. It preserves anonymity due to the fact, that it is not able to deliver high-resolution pictures from faces or license plates, which helps increasing its acceptance. Concerns are often expressed relating to the exposure to electromagnetic fields. The transmit power of a Radar sensor however is limited by regulations to a few milliwatts -very much comparable e.g. to a Bluetooth-signal and is certified to be way below the MPE limits.

\section{Radar Applications and Solutions}

The most important and the simplest feature of a Radar is detecting moving objects and measuring speed very easily taking advantage of the Doppler-Effect. Actually many applications for building technology and home automation require only motion detection. In this market, Radar replaces more and more the Passive Infrared Sensors, which have established in the past because of their low price. Radars however are much more reliable because they are not affected by thermal variations and they offer additional information like direction of movement and the actual speed, making detection safer and more robust. Doppler sensors are used to control lighting, air conditioning, sanitary systems (like toilet flushers and towel dispensers) and for indoor and outdoor building protection.

Automatic doors are nearly always controlled by Radar sensors. It is not only the comfort people enjoy in supermarkets and public buildings, but it is also an aspect of energy saving. Automatic doors help to reduce cost for air conditioning and heating and are therefore a good environmental commitment.

Besides high functionality and reliability, small size, low price and ease of use are very important properties for such kind of sensors. InnoSenT offers an innovative solution with the SMR series. SMR stands for Surface Mountable Radar and describes a tiny, $15 \mathrm{~mm} x$ $20 \mathrm{~mm} \times 3 \mathrm{~mm}$, Radar frontend, which is delivered on tape and reel and which can automatically be pick-and-placed and soldered to the customer's PCB in a standard SMT process. This ultra-small device incorporates a $24 \mathrm{GHz}$ transceiver including low noise amplifier. A person in $10 \mathrm{~m}$ distance can be detected with a signal to noise ratio of more than $20 \mathrm{~dB}$. It consumes only $45 \mathrm{~mA}$ from a $3.3 \mathrm{~V}$ voltage source. For power sensitive applications e.g. in battery powered systems the power consumption can be drastically reduced by operating the sensor in a pulsed mode. Using a duty cycle of $1: 1000$ or even less it is possible to reduce the average operating current to a few microamps. The pulse length can be as short as $1 \mu \mathrm{s}$. Three different antenna variants are available to cover 
beamwidths from $40^{\circ}$ to $120^{\circ}$ degrees. In case, distance information is also necessary, the SMR can be supplied optionally as a VCOtransceiver, which can be frequency-modulated by applying an analog modulation signal to the VCO input. A frequency divider output, switchable between two different division ratios, gives the opportunity to read back the frequency or to operate the device in a phase locked loop.

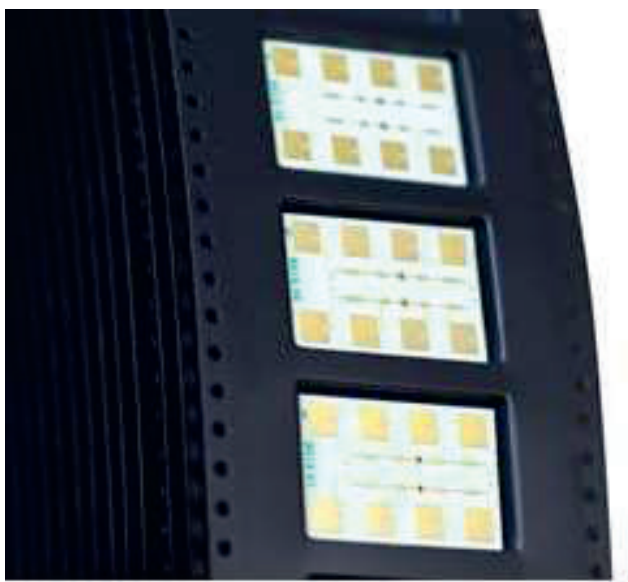

Fig.1. SMR - The Surface Mountable Radar.

Radars are essential also in railway applications.

A True-Speed-Over-Ground sensor is necessary for navigation on every train, because a GPS signal is not always available. In a so-called Janus-configuration, two independent Doppler Radars are arranged in a certain angle and they illuminate the ground from different directions. By correlating both output signals, it is possible to compensate for errors caused by pitching and vibrating.

Railway crossings are usually surveyed by scanning Radars. The high angular resolution, which is necessary to identify objects standing on the track, demands a big aperture antenna like a dish. The Radar is mechanically rotated to cover the region of interest. Subways and urban train systems are more and more using unmanned engines, which require special security measures. InnoSenT provides the sensors for a platform-security-system to survey the tracks in the train station over a length of several hundred meters. Objects of the size of a football lying on the track shall be detected in order to stop the train, smaller objects, e.g. a thrown away beer can shall be ignored. This is realized by a bistatic approach. Pairs of transmitters and receivers facing each other on both sides of the tracks are mounted along the platform. An obstacle lying between transmitter and receiver would attenuate the signal and cause an alarm.

In medical environment, Radar sensors are not yet very common. However, there is an urgent need for a system to detect vital signs like heartbeat or breathing without contact even in some distance.

The contactless monitoring of the vital signs increases the mobility of patients significantly and leads to a better life quality.

Application fields are hospitals and nursing homes, but also the automotive industry is seeking for measures to monitor the driver's health and reactivity, especially in higher automated assistance systems.

A high sensitive Radar is able to detect smallest movements of the skin while penetrating the clothes without significant attenuation. This can be impressively demonstrated on a person who is sitting or lying still, but it is a big challenge to extract the wanted information out of a signal, which is noisy and disturbed by movements of the person and the environment. Evaluations and developments are currently ongoing to overcome this problem.[1]

Collision avoidance is one major task of a Driver Assistance System not only for passenger cars but also in the agricultural and mining environment, and it is an important requirement for highly automated production equipment, robots and logistic vehicles.[2]

Agricultural machines are getting more and more autonomous. Obstacles like fences and stones, but also animals need to be detected in order to ensure secure operation. Because of the dusty environment, Radar is the only reliable solution for securing harvesting machines and controlling seed flow. The huge dimensions of mining vehicles make it impossible for the driver to overview the whole work area without assistance systems. The operation of camera systems is critical because of all the dust and dirt. A Radar based collision avoidance system reduces significantly the risk of accidents.

In industrial production lines, collision avoidance systems are of high importance. In Industry 4.0 the cooperation between man and machine will be enhanced. The robot must be able to detect not only the workpiece it is going to tool, but also obstacles and persons, which are standing or moving within its radius of operation.

Traffic Monitoring is one of the key markets for Radar. Motorways and crossings are often equipped with sensors to observe every lane of 
the street. They are counting cars and classifying vehicles in order to gather statistical data of the traffic load and to regulate the flow of traffic. Classification may be done by measuring the length of the vehicle calculated from the time the object is staying in the Radar beam. The turning wheel delivers a separate line in the Doppler spectrum and -in combination with the distance-informationallows for counting the number of axles of a vehicle.
Traffic Monitoring Radars need to cover a range of 500m and 6 lanes simultaneously. They must be capable of measuring distance, speed and azimuth angle.

In addition, stationary objects must be detected in order to recognize traffic jams or broken down cars on the service lane. Therefore, a simple Doppler-Radar will not do the job.
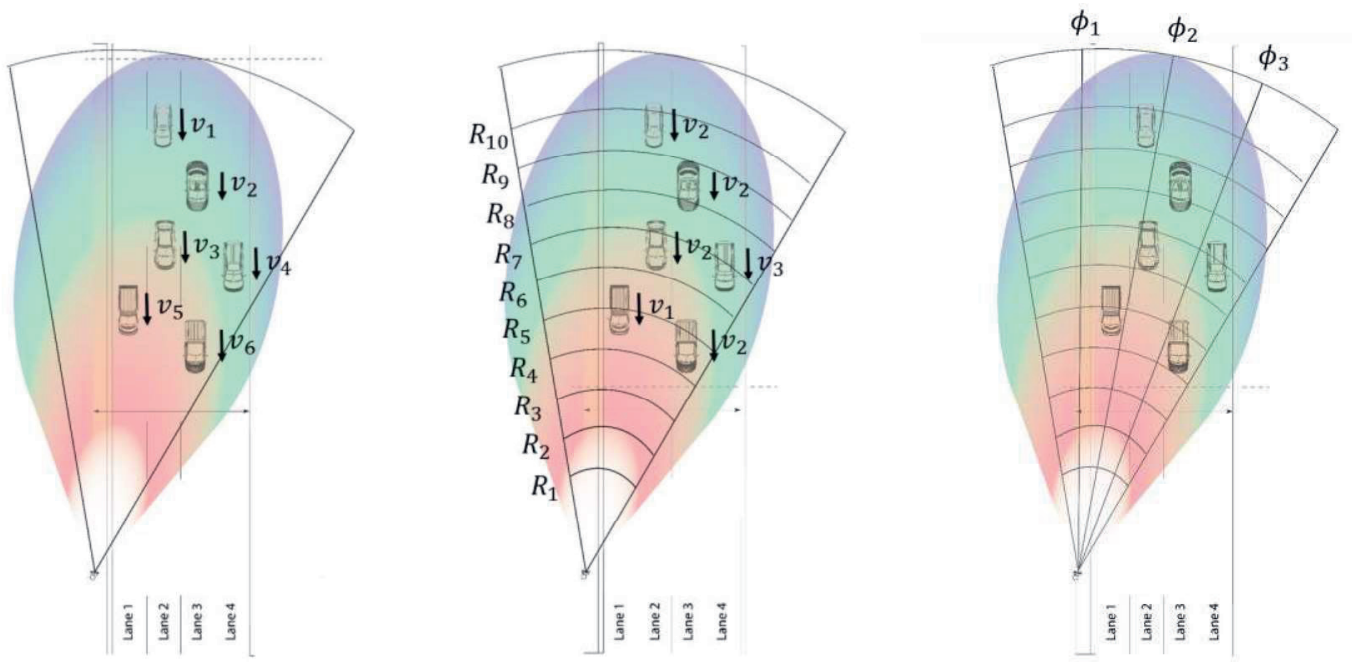

Fig.2. Target Separation in Speed, Distance and Angle.

\section{Modulation Types and Resolution}

Resolution is indeed a very important characteristic of a Radar. Often confused with accuracy, it is actually the ability to separate multiple targets.

An unmodulated Doppler Radar has no resolution at all. Multiple targets can be captured, if they differ in speed, but there is no localization possible. Static objects and radial movement cannot be detected. A FSK-Radar (Frequency Shift Keying) uses the Doppler information on two or more frequencies, which are transmitted alternately. The range information is extracted from the phase shift between the associated Doppler signals. This way target separation in speed and localization in range and angle are possible for objects with different velocity. This type of Radar is well suited for traffic monitoring on motorways and provides velocity resolution of typically $0.5 \mathrm{~km} / \mathrm{h}$. Higher performance and detection of nonmoving objects can be achieved using continuous frequency modulated signals or combined FSK-FMCW (FMSK) modulation. However, it requires more computing effort to separate Doppler and range information and to eliminate unwanted clutter. The range resolution is defined by the modulation bandwidth, which is limited by national regulations. A Radar operating in the $24 \mathrm{GHz}-$ ISM-Band is allowed to cover $250 \mathrm{MHz}$ bandwidth at maximum, which results in a theoretical resolution of $60 \mathrm{~cm}$. This is good enough for a wide range of applications. If higher resolution is required, up to $5 \mathrm{GHz}$ bandwidth is available at $79 \mathrm{GHz}$ for automotive and up to $7 \mathrm{GHz}$ bandwidth at $61 \mathrm{GHz}$ for nonautomotive applications. The angle measurement is realized by using two or more receivers with antennas spaced by typically half a wavelength. The angle information is derived from the phase of the output signals of adjacent receivers. Angular resolution can only be achieved by narrowing down the radiation beamwidth by either physical beamforming (scanning) or digital beamforming. In both cases the resolution is defined by the aperture length, i.e. the effective size of the antenna. Digital beamforming is often used in combination with MIMO architectures (Multiple- 
Input-Multiple-Output) using multiple transmitters and receivers.

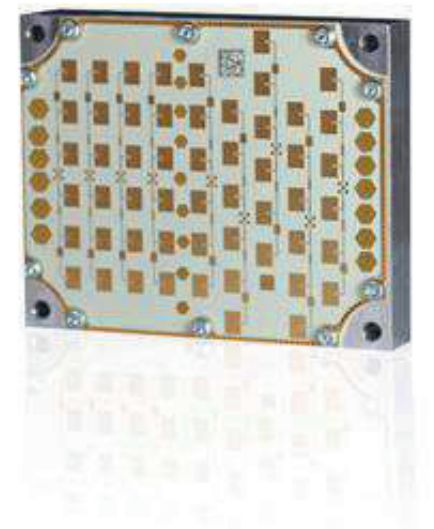

Fig.3. ISYS-5010 - A Digital MIMO Radar.

An example for a sophisticated MIMO Radar is the ISYS-5010. It is a $24 \mathrm{GHz}$-digital beamforming Radar, which uses 4 receivers and two transmitters to generate 8 virtual antennas with an angular resolution of 16 degrees in azimuth. Two additional transmitters are used to extract the angle information in elevation. The Radar employs 2-stage LNAs for each receive channel for lowest noise performance. The distance and speed information are gained from a 2D-Fast-ChirpFMCW modulation. The sensor provides a target list on SPI and it can be configured via UART with the help of a supplied Graphical Users Interface

Fig. 4 shows the modulation scheme of the MIMO-sensor. During a measurement cycle each transmitter generates a chirp sequence of 128 pulses in time multiplex. The duration of a single chirp is typically $12 \mu \mathrm{s}$. A Fast Fourier Analysis is computed for each chirp, gathering the range information, a second FFT across the chirp sequence calculates the speed. The Fast Chirp Modulation results in a high processing gain, which provides an increase in the Signal to Noise Ratio. Another benefit is the fact that the range information is not disturbed by the Doppler shift. Speed and Range are calculated independently.

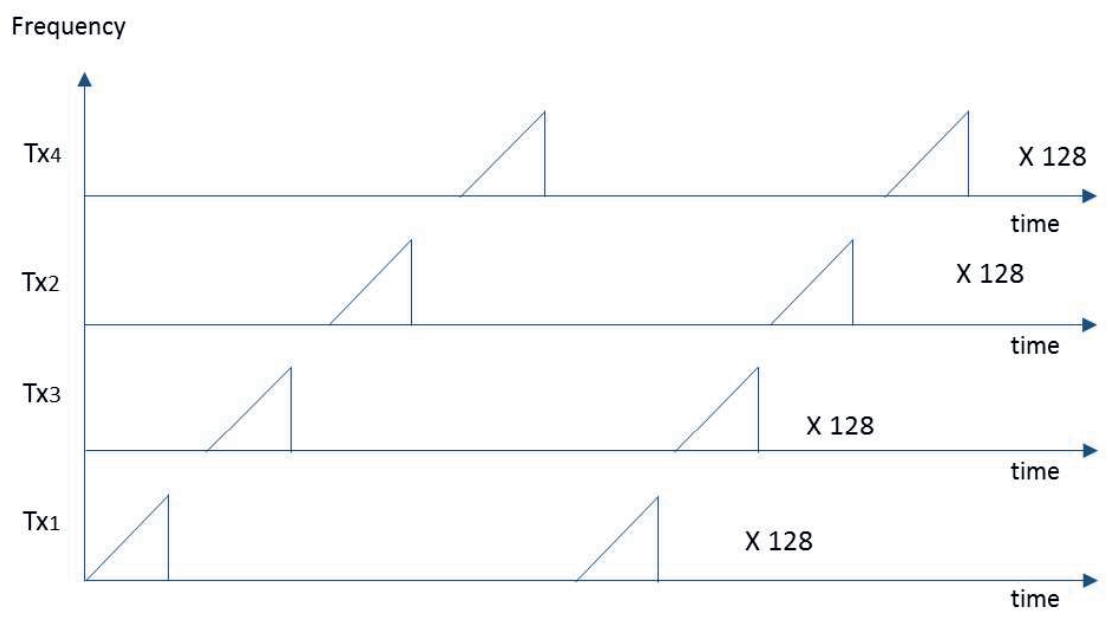

Fig.4. Fast Chirp Modulation in Time Multiplex

\section{Construction and Manufacturing}

The complexity of a Radar sensor varies very much with its application.

A simple analog frontend may consist only of a few components: a small PCB with a printed patch antenna, a PHEM-Transistor and a printed coupling structure forming the oscillator, one or two diodes for the receive mixer and a few resistors and capacitors for DC biasing and decoupling. Such a sensor is produced in millions for ultra-low cost. Going to higher frequencies and more complex systems commercial Microwave Integrated Circuits (MMICs) are used. Fig. 5 shows the component parts of a Digital Sensor. The dimensions of the Radar are almost always determined by the aperture of the antenna, which is unique for each application. The radome is not only protection against mechanical impact, it also 
influences the radiation characteristics decisively and must be considered from the beginning in the antenna design.

Functionality, quality and low cost are the most important attributes, which must be combined in a Radar sensor without any compromise. Low production costs can only be achieved with high volume. Components are getting cheaper with quantity, processes are getting more efficient and tooling costs shrink proportionally. Low production costs also involve high yield, which directly relates to the demand for high quality.

State of the art SMT technology, high precision machines for solder screen printing, placement and soldering as well as quality assurance and inspection equipment, are necessary, but not sufficient. High skilled personnel in engineering, production and quality must permanently improve the products and the processes.

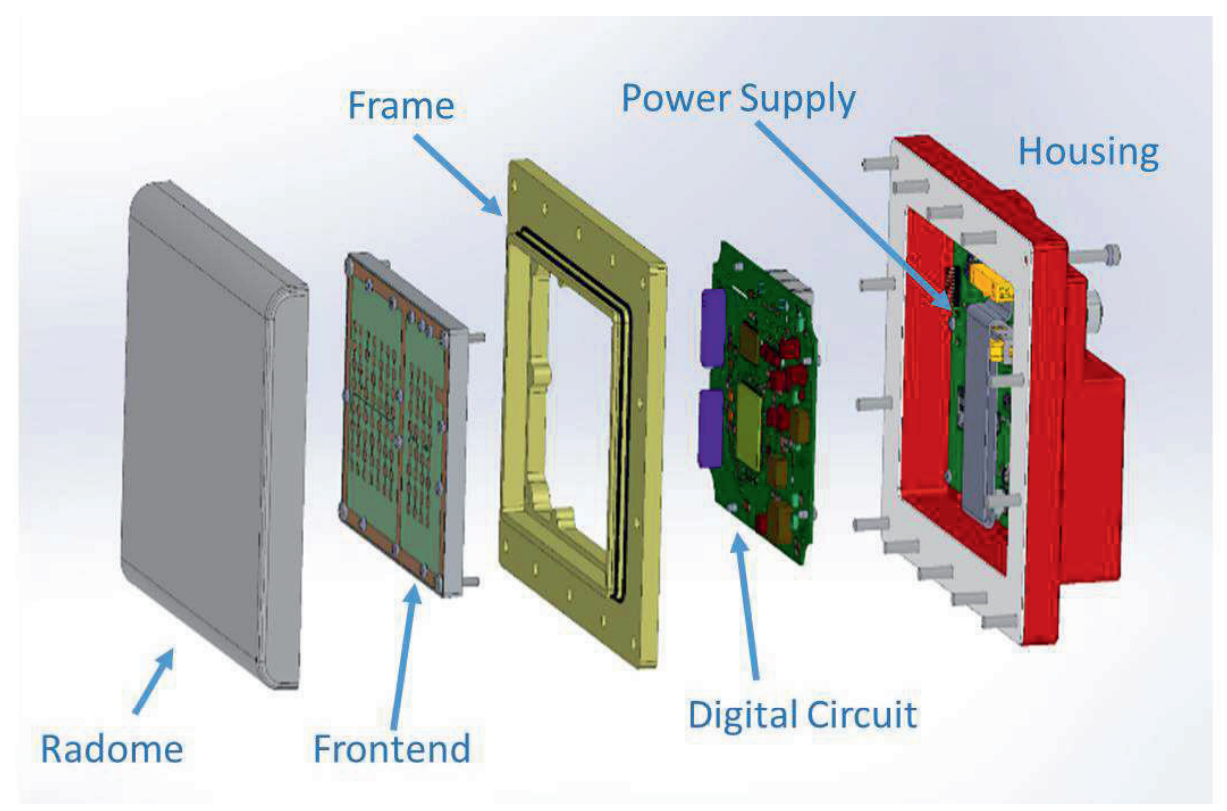

Fig.5. Assembly of a Radar Sensor

\section{Conclusion}

Radar sensors are essential tools for electronic systems providing safety and comfort and are omnipresent in daily life. Very inexpensive solutions are available for presence and motion detectors for smart home applications and intrusion alarm systems. Radars are used for traffic flow regulation, railway security and for distance measurement tasks like tank level probing. The tendency to higher automation, autonomous vehicles and interactive robots requires security systems with $360^{\circ}$ surveillance ability also in adverse environmental conditions where camera systems tend to fail. The increasing demands on reliability and functionality are satisfied by more complex Radar architectures, sophisticated modulation techniques and signal processing, and sufficient computing power. This paper gives an overview over the variety of RADAR applications with different requirements regarding resolution and target separation and shows examples of state of the art commercial RADAR products.

\section{References}

[1] Vinci, G.; Lenhard, T.; Will, C. \& Koelpin, A. Microwave Interferometer Radar-Based Vital Sign Detection for Driver Monitoring Systems IEEE MTT-S International Conference on Microwaves for Intelligent Mobility (ICMIM), 2015

[2] Lenhard T., Kölpin A., "Sichere und interaktive Steuerung von Produktionsanlagen durch vernetzte Umfeldsensoren", MikroSystemTechnik Kongress 2015 